\title{
Immunoglobulin Heavy Chain Variable Domain
}

National Cancer Institute

\section{Source}

National Cancer Institute. Immunoglobulin Heavy Chain Variable Domain. NCI Thesaurus.

Code C129421.

The portion of an antibody molecule that contains the three hypervariable regions in the heavy chains. It is comprised of at most a third of the heavy chains from the amino termini. 